\title{
The COSEHCTM Global Vascular Risk Management quality improvement program: first follow-up report
}

This article was published in the following Dove Press journal:

Vascular Health and Risk Management

19 July 2013

Number of times this article has been viewed

\author{
Carlos M Ferrario' \\ JaNae Joyner ${ }^{2}$ \\ Chris Colby ${ }^{3}$ \\ Alex Exuzides ${ }^{3}$ \\ Michael Moore 2,6 \\ Debra Simmons ${ }^{2}$ \\ William Bestermann $\mathrm{Jr}^{4}$ \\ Feride Frech-Tamas ${ }^{5}$ \\ 'Department of Surgery, Internal \\ Medicine-Nephrology, Wake \\ Forest University School of \\ Medicine, Winston-Salem, NC, USA; \\ ${ }^{2}$ Hypertension and Vascular Research \\ Center, Wake Forest University \\ School of Medicine, Winston-Salem, \\ NC, USA; ${ }^{3} I C O N$ Late Phase and \\ Outcomes Research, San Francisco, \\ CA, USA; ${ }^{4}$ Vascular Medicine Center, \\ Holston Medical Group, Kingsport, \\ TN, USA; ${ }^{5}$ Department of Health \\ Economics, Daiichi Sankyo, Inc, \\ Parsippany, NJ, USA; 'Danville \\ Regional Medical Center, Danville, \\ VA, USA
}

Correspondence: Carlos M Ferrario Wake Forest University School of Medicine, Medical Center Boulevard, Winston-Salem, NC, 27I57, USA

Tel +I 3367169266

Fax +I 3367166644

Email cferrari@wakehealth.edu

\begin{abstract}
The Global Vascular Risk Management (GVRM) Study is a 5-year prospective observational study of 87,863 patients (61\% females) with hypertension and associated cardiovascular risk factors began January 1, 2010. Data are gathered electronically and cardiovascular risk is evaluated using the Consortium for Southeastern Hypertension Control ${ }^{\text {TM }}$ (COSEHC ${ }^{\text {TM}}$ )- 11 risk score. Here, we report the results obtained at the completion of 33 months since study initiation. De-identified electronic medical records of enrolled patients were used to compare clinical indicators, antihypertensive medication usage, and COSEHCTM risk scores across sex and diabetic status subgroups. The results from each subgroup, assessed at baseline and at regular follow-up periods, are reported since the project initiation. Inference testing was performed to look for statistically significant differences between goal attainments rates between sexes. At-goal rates for systolic blood pressure (SBP) were improved during the 33 months of the study, with females achieving higher goal rates when compared to males. On the other hand, at-goal control rates for total and low-density lipoprotein (LDL) cholesterol (chol) were better in males compared to females. Diabetic patients had lower at-goal rates for SBP and triglycerides but higher rates for LDL-chol. The LDL-chol at-goal rates were higher for males, while high-density lipoprotein (HDL)-chol rates were higher for females. Utilization of antihypertensive medications was similar during and after the baseline period for both men and women. Patients taking two or more antihypertensive medications had higher mean COSEHCTM-11 scores compared to those on monotherapy. With treatment, hypertensive patients can reach SBP and cholesterol goals; however, population-wide improvement in treatment goal adherence continues to be a challenge for physicians. The COSEHCTM GVRM Study shows, however, that continuous monitoring and feedback to physicians of accurate longitudinal data is an effective tool in achieving better control rates of cardiovascular risk factors.
\end{abstract}

Keywords: cardiovascular risk, coronary heart disease, dyslipidemia, electronic medical records, hypertension, metabolic syndrome

\section{Introduction}

The metabolic syndrome comprises a constellation of cardiovascular risk factors ${ }^{1,2}$ that include abdominal obesity; insulin resistance; glucose intolerance; elevated blood pressure or antihypertensive drug treatment; low levels of high-density lipoprotein (HDL) cholesterol (chol); and elevated triglyceride levels. Abundant evidence shows that the metabolic syndrome predicts the development of cardiovascular disease (CVD). ${ }^{3-5}$ In a previous article, ${ }^{6}$ we presented the baseline demographic profile and risk factor prevalence among patients enrolled in an observational large scale assessment of cardiometabolic risk factors in the southeastern United States, a region in which 
the Consortium for Southeastern Hypertension Control ${ }^{\mathrm{TM}}$ (COSEHC ${ }^{\mathrm{TM}}$ ) was among the first to raise awareness of an excess in cardiovascular-related deaths. ${ }^{6-15}$

The Global Vascular Risk Management (GVRM) Study is a large-scale project to prospectively determine the sensitivity of the COSEHC-cardiovascular risk assessment tool (COSEHC-CRT) to provide benchmarking data on treatment patterns and outcomes to participating COSEHC-designated Cardiovascular Centers of Excellence ${ }^{\mathrm{TM}}{ }^{6}$ De-identified electronic medical records were used to compare clinical indicators, antihypertensive medication usage, and COSEHC ${ }^{\text {TM }}$ risk scores across sex and diabetic status subgroups. In this article, we examined the changes in cardiovascular risk factors and rate of clinical events for those patients who continued care with COSEHC ${ }^{\text {тм }}$ health care providers during the first 33 months follow-up period from the reported baseline. ${ }^{6}$ The report documents the changes in systolic blood pressure (SBP), cholesterol measures, and body mass index (BMI) across sex and diabetic status subgroups. We also present results on the utilization of antihypertensive medications for treatment of hypertension, lipid management, and diabetes, along with an evaluation of the COSEHC-CRT score as a function of antihypertensive medication usage. ${ }^{13}$ The information, gathered throughout the first 33 months of the study, provides new information as to how a cohort of subjects from a population with a documented high rate of cardiovascular events and the participating physicians respond to a structured longitudinal surveillance process based on assessment of clinical and therapeutic goals through the use of electronic medical records (EMRs). The information attained in this study may be used by physicians to understand the value of EMRs and have increased awareness of expected results in terms of sex, clustering of risk factors including diabetes, and therapeutic effectiveness.

\section{Methods}

\section{Study design}

The GVRM Study is a voluntary, observational, prospective quality-improvement initiative conducted at eight COSEHCTM-designated Cardiovascular Centers of Excellence $^{\mathrm{TM}}$ from patients who were at least 18 years of age and met one or more of the following criteria: (1) at least one predefined International Classification of Disease, 9th Revision (ICD-9) CVD risk factor code; (2) treatment at a participating COSEHC ${ }^{\text {TM}}$-designated Center of Excellence; and (3) alternative treatment at a noncardiac-related outpatient clinic for a cardiac-related Current Procedural Terminology code condition. All patients were treated per the standard of care for their respective conditions, and no predefined visits, medical, laboratory tests, procedures, or interventions were required. Of the eleven variables in the modified COSEHCCRT score ${ }^{6}$ age, sex, smoking status, and family history of coronary heart disease (CHD) were collected only once, all other data were updated on a quarterly basis.

\section{Study duration and outcomes}

The baseline measures for this 5-year prospective study are published elsewhere. ${ }^{6}$ The follow-up time period analyzed in this article is 33 months (January 1, 2010, to September 30, 2012).

\section{Data flow and management}

The GVRM Study was approved on a central level by the Copernicus Group Institutional Review Board (IRB) (Research Triangle Park, Durham, NC, USA) and by the IRBs of each participating site. The selected numerical values from individual patient EMRs were submitted to the COSEHCTM Coordinating Center at the Wake Forest University School of Medicine on a quarterly basis. The following ICD-9 codes were used for clinical events: anterior myocardial infarction (AMI: 410); acute coronary syndrome (ACS: 411); stroke: 430-434; transient ischemic attack (TIA: 435); other cardiovascular disease (CVD: 436); and congestive heart failure (CHF: 428). Only patients with no evidence of the specified condition during the baseline period, and a subsequent diagnosis for that condition during follow-up are included in the subanalysis. Antihypertensive medication usage was defined as number of refills (see Statistical methods).

\section{Statistical methods}

The analysis focused on patients from the study baseline period who continued care with COSEHCTM health care providers during the follow-up period (at least one visit throughout each of the following years). We evaluated clinical measures and treatment goal attainment across patient subgroups defined by sex and diabetic status, as recorded in Table 1. At-goal rates, defined as achieving current clinical practice guidelines ${ }^{16,17}$ were: hypertension $(\mathrm{SBP}<140 \mathrm{mmHg}$ for nondiabetic subjects and $<130 \mathrm{mmHg}$ for those with an associated diagnosis of chronic kidney disease or diabetes); total cholesterol $<200 \mathrm{mg} / \mathrm{dL}$, HDL-chol $>40 \mathrm{mg} / \mathrm{dL}$, lowdensity lipoprotein (LDL)-chol $<100 \mathrm{mg} / \mathrm{dL}$; and plasma triglycerides $<150 \mathrm{mg} / \mathrm{dL}$. These goal attainment rates were also compared from the baseline period to the follow-up period. 
Table I Average differences in goal achievement at the end of 33 months follow-up

\begin{tabular}{|c|c|c|c|c|c|}
\hline \multirow[t]{2}{*}{ Variable and condition } & \multicolumn{2}{|l|}{ Females } & \multicolumn{2}{|l|}{ Males } & \multirow[t]{2}{*}{$P$-value ${ }^{t \dagger}$} \\
\hline & $M \pm S D(N)$ & $\begin{array}{l}\text { At-goal } \\
\text { rate }\end{array}$ & $\mathbf{M} \pm \mathrm{SD}(\mathrm{N})$ & $\begin{array}{l}\text { At-goal } \\
\text { rate }\end{array}$ & \\
\hline \multicolumn{6}{|l|}{ All subjects } \\
\hline \multicolumn{6}{|c|}{ Systolic blood pressure, $\mathrm{mmHg}$} \\
\hline Baseline values $^{\dagger}$ & $128 \pm 17(53,025)$ & $68 \%$ & $130 \pm 17(33,762)$ & $62 \%$ & $<0.0001$ \\
\hline Follow-up values ${ }^{\ddagger}$ & $128 \pm 17(5 \mid, 368)$ & $70 \% * *$ & $129 \pm 17(32,770)$ & $65 \% * *$ & $<0.000 \mathrm{I}$ \\
\hline \multicolumn{6}{|l|}{ Total cholesterol, mg/dL } \\
\hline Baseline values $^{\dagger}$ & $|90 \pm 4|(53,589)$ & $62 \%$ & $170 \pm 40(34,209)$ & $79 \%$ & $<0.0001$ \\
\hline Follow-up values ${ }^{\ddagger}$ & $|86 \pm 4|(33,809)$ & $66 \% * *$ & $164 \pm 39(24,039)$ & $83 \% * *$ & $<0.0001$ \\
\hline \multicolumn{6}{|l|}{ LDL-cholesterol, mg/dL } \\
\hline Baseline values $^{\dagger}$ & III $\pm 35(53,308)$ & $40 \%$ & $99 \pm 34(34,037)$ & $54 \%$ & $<0.0001$ \\
\hline Follow-up values ${ }^{\ddagger}$ & $106 \pm 35(32,502)$ & $46 \% * *$ & $93 \pm 32(23,294)$ & $62 \% * *$ & $<0.0001$ \\
\hline \multicolumn{6}{|l|}{ HDL-cholesterol, mg/dL } \\
\hline Baseline values $^{\dagger}$ & $52 \pm 15(53,570)$ & $78 \%$ & $42 \pm 12(34,204)$ & $47 \%$ & $<0.0001$ \\
\hline Follow-up values ${ }^{\ddagger}$ & $53 \pm 15(33,705)$ & $80 \% * *$ & $42 \pm 12(23,980)$ & $50 \% * *$ & $<0.0001$ \\
\hline \multicolumn{6}{|l|}{ Plasma triglycerides, $\mathrm{mg} / \mathrm{dL}$} \\
\hline Baseline values $^{\dagger}$ & $136 \pm 82(53,19 \mid)$ & $68 \%$ & $147 \pm 93(33,6 \mid 4)$ & $63 \%$ & $<0.0001$ \\
\hline Follow-up values $s^{\ddagger}$ & $137 \pm 83(33,719)$ & $67 \% * *$ & $144 \pm 94(23,975)$ & $65 \% * *$ & $<0.000$ I \\
\hline \multicolumn{6}{|l|}{ Nondiabetic patients } \\
\hline \multicolumn{6}{|c|}{ Systolic blood pressure, $\mathrm{mmHg}$} \\
\hline Baseline values $^{\dagger}$ & $127 \pm 17(35,8 \mid 5)$ & $78 \%$ & $130 \pm 16(18,510)$ & $73 \%$ & $<0.0001$ \\
\hline Follow-up values ${ }^{\ddagger}$ & $127 \pm 17(34,593)$ & $79 \% * *$ & $128 \pm 16(17,971)$ & $76 \% * *$ & $<0.0001$ \\
\hline \multicolumn{6}{|l|}{ Total cholesterol, mg/dL } \\
\hline Baseline values $^{\dagger}$ & $193 \pm 39(36,125)$ & $60 \%$ & $174 \pm 39(18,744)$ & $76 \%$ & $<0.0001$ \\
\hline Follow-up values ${ }^{\ddagger}$ & $190 \pm 39(21,219)$ & $63 \% * *$ & $169 \pm 37(12,570)$ & $81 \% * *$ & $<0.0001$ \\
\hline \multicolumn{6}{|l|}{ LDL-cholesterol, mg/dL } \\
\hline Baseline values $^{\dagger}$ & $114 \pm 34(36,072)$ & $36 \%$ & $104 \pm 34(18,709)$ & $48 \%$ & $<0.0001$ \\
\hline Follow-up values ${ }^{\ddagger}$ & $110 \pm 34(20,193)$ & $41 \% * *$ & $98 \pm 32(12,066)$ & $56 \% * *$ & $<0.0001$ \\
\hline \multicolumn{6}{|l|}{ HDL-cholesterol, mg/dL } \\
\hline Baseline values $^{\dagger}$ & $54 \pm 16(36,122)$ & $82 \%$ & $43 \pm 13(18,738)$ & $53 \%$ & $<0.0001$ \\
\hline Follow-up values ${ }^{\ddagger}$ & $55 \pm 15(21,158)$ & $85 \% * *$ & $45 \pm 12(12,540)$ & $57 \% * *$ & $<0.0001$ \\
\hline \multicolumn{6}{|l|}{ Plasma triglycerides, $\mathrm{mg} / \mathrm{dL}$} \\
\hline Baseline values $^{\dagger}$ & $|25 \pm 7|(36,089)$ & $73 \%$ & $|35 \pm 8|(\mid 8,692)$ & $69 \%$ & $<0.0001$ \\
\hline Follow-up values ${ }^{\ddagger}$ & $125 \pm 72(21,142)$ & $73 \% * *$ & $|3| \pm 8 \mid(\mid 2,532)$ & $71 \% * *$ & $<0.0001$ \\
\hline \multicolumn{6}{|l|}{ Diabetic subjects } \\
\hline \multicolumn{6}{|c|}{ Systolic blood pressure, $\mathrm{mmHg}$} \\
\hline Baseline values $^{\dagger}$ & $|3| \pm 18(|7,2| 0)$ & $48 \%$ & $130 \pm 17(15,252)$ & $49 \%$ & 0.1926 \\
\hline Follow-up values ${ }^{\ddagger}$ & $130 \pm 18(16,775)$ & $51 \% * *$ & $129 \pm 17(14,799)$ & $51 \% * *$ & 0.5068 \\
\hline \multicolumn{6}{|l|}{ Total cholesterol, mg/dL } \\
\hline Baseline values $^{\dagger}$ & $184 \pm 43(17,464)$ & $68 \%$ & $|65 \pm 4|(15,465)$ & $82 \%$ & $<0.0001$ \\
\hline Follow-up values ${ }^{\ddagger}$ & $180 \pm 44(12,590)$ & $72 \% * *$ & $160 \pm 40(11,469)$ & $86 \% * *$ & $<0.0001$ \\
\hline \multicolumn{6}{|l|}{ LDL-cholesterol, mg/dL } \\
\hline Baseline values $^{\dagger}$ & $104 \pm 36(17,236)$ & $49 \%$ & $94 \pm 33(15,328)$ & $62 \%$ & $<0.0001$ \\
\hline Follow-up values ${ }^{\ddagger}$ & $100 \pm 36(12,309)$ & $55 \% * *$ & $89 \pm 33(11,228)$ & $69 \% * *$ & $<0.0001$ \\
\hline \multicolumn{6}{|l|}{ HDL-cholesterol, mg/dL } \\
\hline Baseline values $^{\dagger}$ & $48 \pm 14(17,448)$ & $71 \%$ & $40 \pm I I(15,466)$ & $40 \%$ & $<0.0001$ \\
\hline Follow-up values ${ }^{\ddagger}$ & $49 \pm 13(12,547)$ & $72 \% * *$ & $40 \pm 11(11,440)$ & $41 \% * *$ & $<0.0001$ \\
\hline Plasma triglycerides, $\mathrm{mg} / \mathrm{dL}$ & & & & & \\
\hline Baseline values $^{\dagger}$ & $159 \pm 95(17,102)$ & $57 \%$ & $162 \pm 103(14,922)$ & $57 \%$ & 0.2126 \\
\hline Follow-up values ${ }^{\ddagger}$ & $157 \pm 96(12,577)$ & $58 \% * *$ & $158 \pm 104(\mid \mathrm{I}, 443)$ & $59 \% * *$ & 0.3650 \\
\hline
\end{tabular}

Notes: Values are means \pm I standard deviation $\left(M \pm S D\right.$ ) of variables obtained before (baseline) and at the end of the 4th quarter in 2012 (follow-up values). ${ }^{\dagger}$ Includes all patients with at least 8/II COSEHC TM variables observed during the baseline time period, and at least one visit during the 20I0-QI-20I2-Q4 time period; \#includes all patients with at least 8/II COSEHC TM variables observed during the baseline time period, and observation for that measure during the 20I0-QI-20I2-Q4 time period. Because some patients had missing at-goal values, Row 3 and Row 4 do not necessarily add up to Row 2 numbers; **indicates a statistically significant difference (Chi-square test) from baseline to follow-up with $P$-value $<0.0001$; $+1 P$-value for the difference between male and female at-goal rates for each measure.

Abbreviations: M, mean; N, number; SD, standard deviation; COSEHC, Consortium for Southeastern Hypertension Control; HDL, high-density lipoprotein; LDL, low-density lipoprotein; Q, quarter. 
We used the Chi-square test to compare at-goal rates. In addition to these clinical measures, antihypertensive medication usage, BMI, and the COSEHCTM score were also compared across patient subgroups.

As this is an observational study not all patients received every lab test during the follow-up period, and some patients had more than one value for some risk factors. Thus, the sample size differed across all measures. When patients had more than one value, the most recent valid follow-up value was used. When patients had only a subset of the measures recorded during follow-up, the patient remained in the study. There was no carry-forward of values from the baseline period other than the patient's height for computation of BMI.

A separate analysis for the diabetic subpopulation (by sex) was also performed. The percentage of patients at-goal for each measure was calculated. These percentages were calculated for both the baseline and follow-up period, with separate reporting for patients who were or were not at-goal at baseline. The total number of antihypertensive medications either prescribed during the follow-up period or continued from the baseline period was counted for each patient. All unique antihypertensive medication names were used as the basis for these counts. Since this is a count of prescriptions written, it does not distinguish between a patient switching medications during follow-up or concurrently adding a second medication.

As documented by us previously, ${ }^{6,13}$ the COSEHC-CRT score is an additive score calculated with separate algorithms for men and women. The individual scoring elements are: age (5-year ranges); smoking status (with differential scores for each age range); SBP ranges; laboratory test score ranges; and patient history variables. The final COSEHC ${ }^{\mathbf{T M}}$ score is the sum of each of these individual scoring elements. We also report treatment goal attainment rates for each CVD factor by sex and diabetic status. These at-goal rates were reported for baseline and follow-up (with separate reporting for patients not at-goal during baseline and patients at-goal during baseline). Inference testing was performed to look for statistically significant differences between goal attainment rates by sex. SBP at-goal status was modeled as a function of sex, clinical history, and follow-up at-goal status for the following measures (LDL-chol, HDL-chol, triglycerides, and BMI). Logistic regression using SAS/STAT software, (Version 9.2 of the $\mathrm{SAS}^{\circledR}$ System for Windows. Copyright 2002-2008 SAS Institute, Cary, NC, USA) was also performed, modeling the at-goal rate for SBP during follow-up.
This model was developed to determine correlation between baseline risk factors and SBP at-goal rates. Independent variables included sex, risk factors such as smoking and diabetic status, and adherence to other treatment goals, including HDL-chol, LDL-chol, triglycerides, and BMI.

\section{Results \\ Demographic and clinical history}

Out of the 87,863 patients with at least one follow-up visit, $61 \%$ were female (mean age of 62 years). Of the 76,383 patients with known race/ethnicity, $15.1 \%$ were African-Americans; $77.7 \%$ were Caucasian; less than $1 \%$ were Hispanic, and $6.6 \%$ were listed as other. According to medical record review, $32.2 \%$ of the patients were current smokers, $38.4 \%$ were diabetics, $2.4 \%$ reported a history of premature CHD in their family, and $3.8 \%$ had left ventricular hypertrophy.

Table 1 shows the group averages of systolic blood pressures and lipids at 33 months of follow-up and the changes in at-goal achievement rates for the entire patient cohort and the subgroups of subjects who did or did not have a diabetic diagnosis at the time of patient enrollment.

\section{Systolic blood pressure}

At the time of enrollment (baseline; Table 1), goal-attainment rates for SBP in the entire population are above $60 \%$. The highest goal attainment rates at baseline are found in the subgroup of subjects with a negative history of diabetes (78\%; Table 1), while the lowest at-goal rates are present in diabetic subjects (48\%; Table 1). Modest, albeit statistically significant, improvements in SBP at-goal rates were observed in all subjects at the completion of the follow-up period (Table 1). Improvement in the at-goal rates for diabetic subjects $(51 \%$; Table 1$)$ is significantly lower $(P<0.05)$ than those measured for the entire population $(70 \%)$ or the subgroups of subjects with a negative diagnosis of diabetes at baseline (79\%). Table 1 also shows lower SBP values and higher at-goal rates for males compared to females for the whole patient population and nondiabetic subjects. In contrast, SBP at-goal rates in diabetic subjects are not different in males and females (Table 1).

\section{Lipid profile}

Baseline at-goal rates for total-chol, HDL-chol, and triglycerides are greater than $50 \%$ at the time of patient enrollment into the study (Table 1). Overall, LDL-cholesterol baseline at-goal rates are below 50\% (Table 1). Over the course of the 33 months of follow-up, small improvements in lipid 
at-goal rates are present for both nondiabetic and diabetic patients (Table 1). In addition, at-goal rates for total-chol and LDL-chol are significantly higher in males compared to females whether comparisons are made for all subjects or those without diabetes. At-goal rates improved from baseline to follow-up for males and females in both subgroups $(P<0.0001)$, with an overall improvement in the total-chol rate from $62 \%$ to $66 \%$ for males and $79 \%$ to $83 \%$ for females (Table 1). These findings contrast with the concurrent demonstration of lower at-goal rates for LDL-chol when compared to total-chol for all subjects or the subgroup without diabetes (Table 1). The conversion rate for baseline not-at-goal patients and the retention rate for patients at-goal during baseline rate were also lower for diabetic patients.

Total-chol at-goal rates for subjects that were at-goal at baseline were higher for males than females (66\% versus $83 \% ; P<0.0001$ ) during the follow-up period (Table 1 , all subjects). Goal-retention rates for patients at-goal for LDL-chol during baseline were higher for males compared to females, and this sex difference persisted throughout the follow-up period (Table 1). In terms of HDL measurements, lower values of HDL-chol at-goal rates for both sexes showed significantly lower at-goal rates in males, not at baseline goal. This difference, albeit attenuated in magnitude, is still present in males who were at-goal for HDL-chol (Figure 1). For plasma triglycerides, diabetic females and males had similar goal rates for all cohorts. These rates were also similar when compared to those for all patients with the exception of the conversion rate among diabetic males that was higher than that for all male patients (Figure 1). For the BMI measure, we observed lower rates across all cohorts, including the conversion rate for baseline at-goal patients and the retaining rate for patients at-goal during baseline, when compared to all patients.

\section{Influence of diabetic status}

Figure 1 shows the follow-up results for subjects who were or were not at-goal at baseline in diabetic and nondiabetic subjects. It is evident that being not at-goal at the initiation of the study is associated with similar, lesser attainments of goal rates during the follow-up period. Within the diabetic subgroup, both females and males had lower at-goal rates for SBP during the follow-up period, as well as lower conversion rates when compared to the entire cohort of patients (Figure 1 and Table 1). This is primarily due to the stricter goal criteria for diabetic patients $(<130 \mathrm{mmHg}$ for diabetics versus $<140 \mathrm{mmHg}$ for nondiabetics without chronic kidney disease).

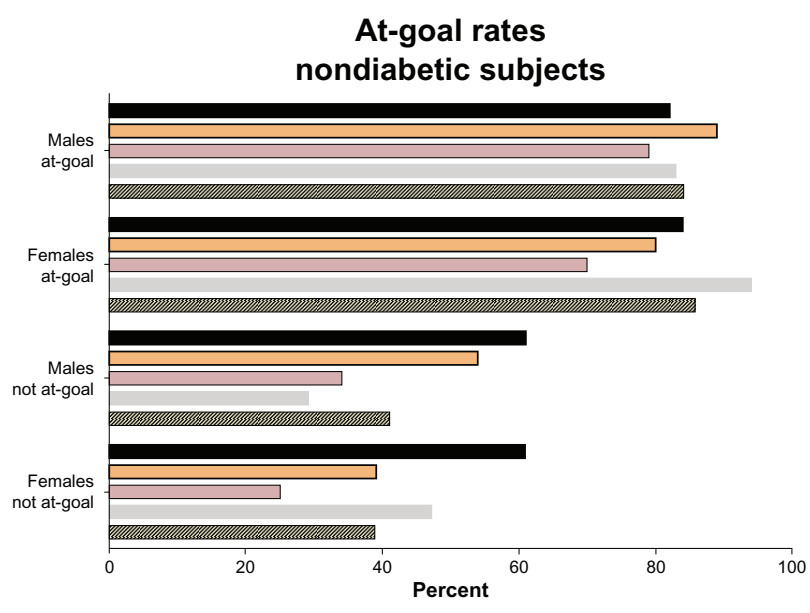

- SBP $\square$ T-cholesterol $\square$ LDL-cholesterol $\square$ HDL-cholesterol $\mathbb{Z}$ Triglycerides

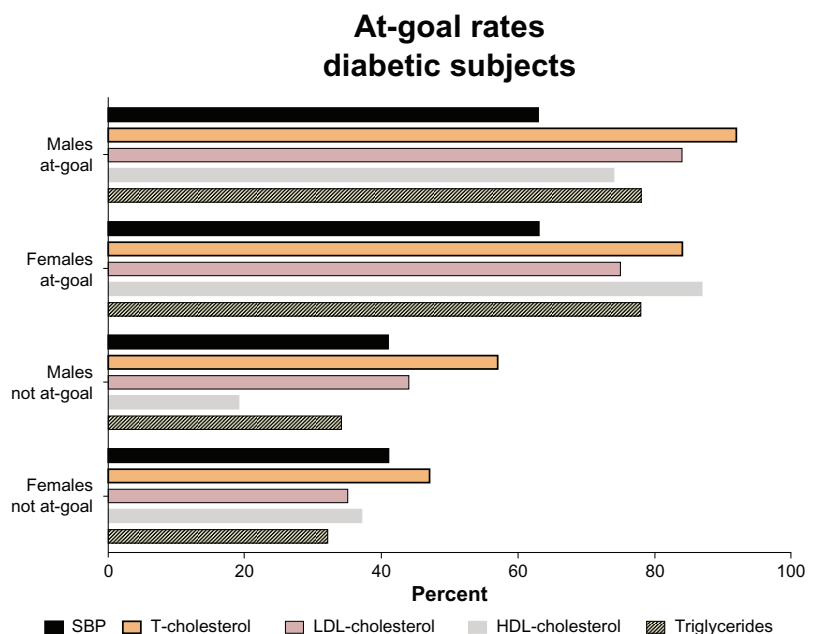

Figure I Attainment of at-goal rates at the completion of the 33 months for subjects who were at-goal versus not at-goal for the measured cardiovascular risk factors. Abbreviations: SBP, systolic blood pressure; LDL, low-density lipoprotein; HDL, high-density lipoprotein; T, total.

\section{COSEHC-CRT score}

Figure 2 shows higher COSEHC-CRT scores in males at the initiation of the study, at follow-up, and even in the subset of subjects who were not at-goal during the baseline period and whether they are being treated with one or more antihypertensive drugs. Higher COSEHC-CRT score values in males, in part, reflects the fact that the COSEHCTM score itself automatically assigns higher scores for males than females. ${ }^{6,13}$

Utilization of antihypertensive medications was similar in the at-goal patient population for both men and women $(P>0.05)$; however, the not-at-goal female subgroup had slightly higher average prescription counts compared to the male subgroup (2.2 versus $2.1 ; P<0.0001)$. As illustrated in Figure 2, patients taking two or more antihypertensive 

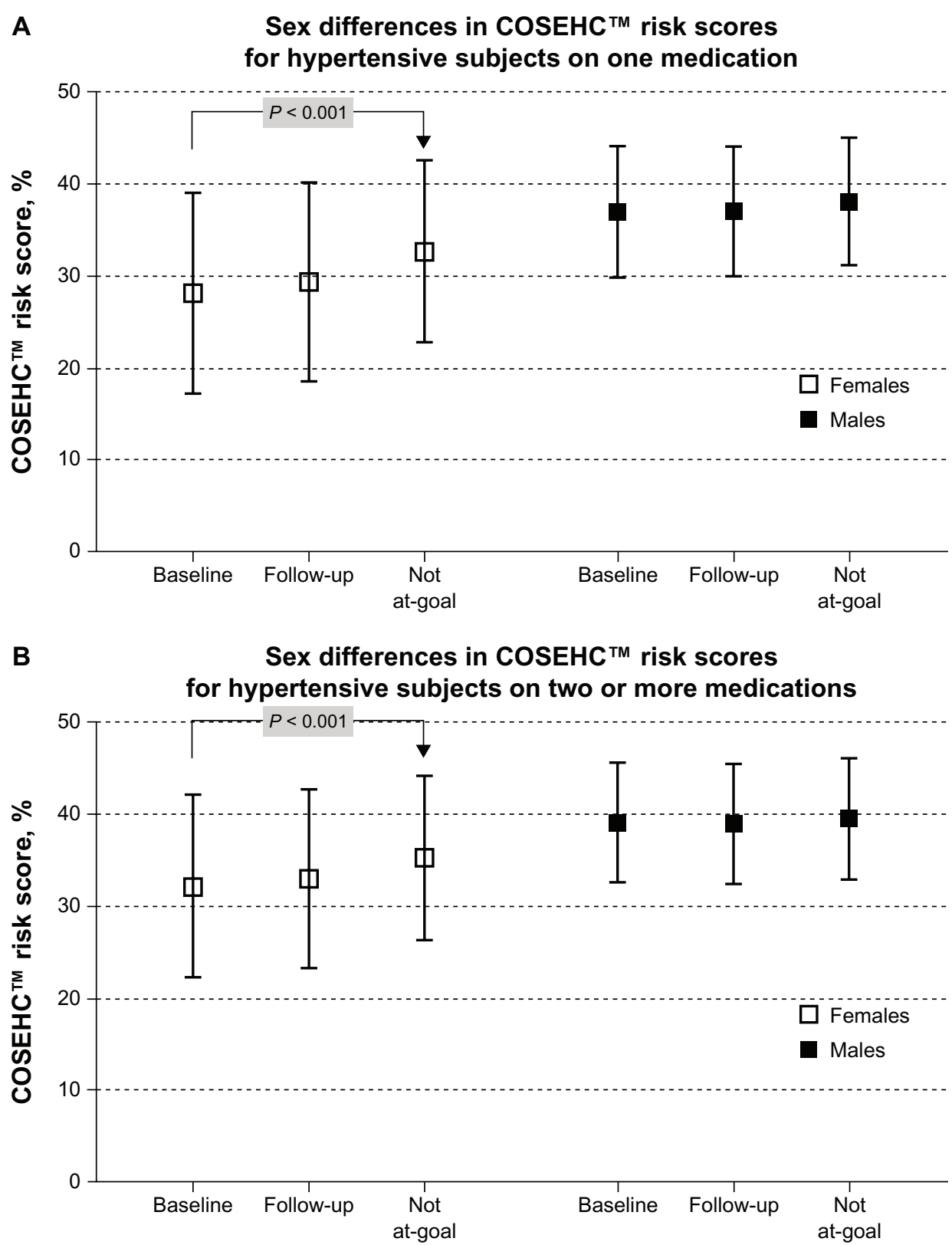

Figure 2 Sex differences in COSEHC TM risk scores.

Notes: Although sex has a direct influence on the Consortium for Southeastern Hypertension Control ${ }^{\mathrm{TM}}$ (COSEHC) risk factor scoring, the data show a significant increase in the risk for females who are not at-goal at the end of the examined period. Values are means \pm standard deviation of the risk score for males and females with one (A) or two or more (B) medications. Statistical differences are denoted in the graph. All other comparisons were not statistically significant $(P>0.05)$.

medications had higher mean COSEHC-CRT scores for both females and males, compared to those taking a single antihypertensive medication.

A logistic regression analysis was performed to model the probability of SBP at-goal status during follow-up as a function of sex, diabetic status, and other measures. Diabetes, smoking status, and BMI were significant at $P<0.05$. Diabetes status had the smallest odds ratio $(\mathrm{OR}=0.36)$ and was significant at $P<0.0001$, showing that diabetics were at least three times more likely to not be at-goal for SBP. In other words, the logistic regression analysis indicates that being at-goal for SBP is more likely if the subject is a nonsmoker who is at-goal for both BMI and LDL-cholesterol and is nondiabetic. The Hosmer-Lemeshow Goodness of Fit Test had a $P$-value of 0.2578 . This high $P$-value indicates a good model fit.

Figure 3 shows the number of clinical events occurring throughout the follow-up period (2010-2012). There were a total of 832 clinical events among 811 patients $(0.92 \%$ of the total patient population). Strokes and CHF represented the highest event categories. 


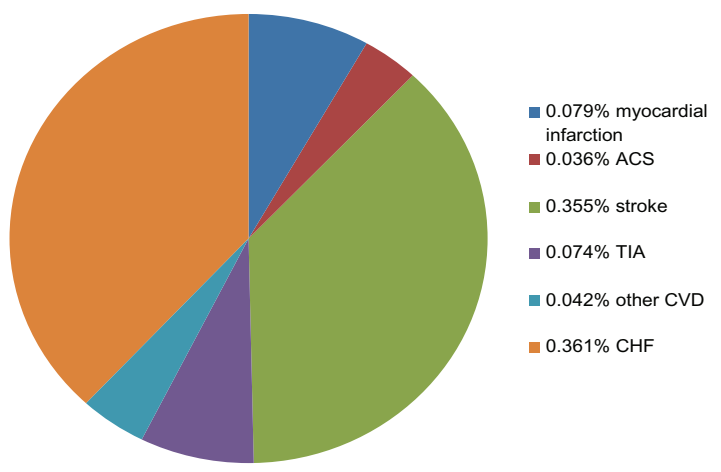

Figure 3 Clinical events documented during the 33 months for the overall patient population.

Notes: Data are from events recorded by physicians using International Classification of Disease, 9th Revision (ICD-9) codes starting with 4I0 (see Methods). Stroke includes both hemorrhagic and ischemic stroke.

Abbreviation: ACS, acute coronary syndrome; TIA, transient ischemic attack; $\mathrm{CHF}$, congestive heart failure; CVD, other cardiovascular events.

\section{Discussion}

Multiple theories have been suggested to address the factors contributing to poor medication compliance, quality of care, and patient outcomes. The results obtained during a 33 month follow-up of a large practice-based southeastern high cardiovascular risk patient population observational study show improvements in blood pressure control, as well as modest increases in the at-goal rates for total cholesterol, LDL-chol, and HDL-chol that are most evident in males compared to females. Presence of diabetes mellitus worsens the ability of subjects to reach or maintain cardiovascular risk factors' at-goal control rates while the data also confirm the higher risk that males are exposed to compared to females in terms of cardiovascular events, as reflected in the higher COSEHC-CRT score rates.

The GVRM Study seeks to provide prospective benchmarking of recognized cardiovascular risk factors on treatment patterns and patient outcomes from eight of 33 COSEHCTM designated Cardiovascular Centers of Excellence ${ }^{\mathrm{TM}}$. The population served by the physicians enrolled in the study includes a large number of patients at high risk for cardiovascular events due to their location in the southeastern USA, a region with a recognized higher cardiovascular morbidity and mortality. ${ }^{8,10,12,14,15,18-20}$ Data, appropriately de-identified, were collected electronically from each participating center, and the subjects included in the study met the inclusion criterions defined in our previous publication. ${ }^{6}$ Importantly, the current report provides a glimpse of the study's predefined main outcome measures of cardiovascular morbidity and mortality, including the development of CHD, CHF, stroke, transient ischemic attack, abdominal aortic aneurysm, myocardial infarction, renal failure, and diabetic retinopathy.

The overall number of clinical events, approximately $0.92 \%$ of the total monitored population over the 33 month observational period, is relatively low as the at-goal rates were generally higher than $50 \%$, except in diabetic subjects. Since the COSEHC-CRT score estimates the 5-year absolute cardiovascular mortality risk, and individuals can be identified as high relative risk ( $>60$ th percentile) compared to the reference population, or identified as high absolute risk (5-year mortality risk exceeding $2.5 \%$ ), the average $32 \%-36 \%$ score measured in our population indicates a higher than $0.85 \%$ risk of 5 -year cardiovascular mortality. ${ }^{13}$ This finding suggests a lower occurrence of events compared with other studies having similar commonalities in terms of patient characteristics and follow-up. ${ }^{21-24}$ In assessing the benefits of a healthy diet on the composite of cardiovascular events (cardiovascular death (CV); myocardial infarction (MI); stroke; or CHF) in the combined patient population enrolled in the Ongoing Telmisartan Alone in Combination with Ramipril Global End Point Trial (ONTARGET; 4,221 patients) and in the Telmisartan Randomized Assessment Study in ACEI Intolerant Subjects with Cardiovascular Disease (TRANSCEND; 969 patients) trials, ${ }^{25-30}$ Dehghan et $\mathrm{al}^{23}$ showed that the rates of clinical events for myocardial infarction, stroke, and congestive heart failure in their population are higher than those found in our study. These data

Table 2 Logistic regression results

\begin{tabular}{|c|c|c|c|c|}
\hline Effect & Odds ratio & \multicolumn{2}{|c|}{ 95\% Wald confidence limits } & $P$-value \\
\hline \multicolumn{5}{|c|}{ Dependent variable: follow-up SBP at-goal } \\
\hline Sex: female & 0.97 & 0.89 & 1.06 & NS \\
\hline Presence of diabetes & 0.36 & 0.31 & 0.42 & $<0.0001$ \\
\hline LDL-cholesterol at-goal & 1.12 & 1.03 & 1.23 & 0.0087 \\
\hline HDL-cholesterol at-goal & 0.92 & 0.83 & 1.01 & NS \\
\hline Plasma triglycerides at-goal & $\mathrm{I} .07$ & 0.98 & 1.17 & NS \\
\hline Smoking status: nonsmoker & 0.82 & 0.75 & 0.89 & $<0.0001$ \\
\hline Body mass index at-goal & 1.33 & 1.18 & $|.5|$ & $<0.0001$ \\
\hline
\end{tabular}

Notes: $\mathrm{N}=9,377 ; 5,180$ at SBP goal; 4,197 not at SBP goal.

Abbreviations: SBP, systolic blood pressure; NS, not significant; HDL, high-density lipoprotein; LDL, low-density lipoprotein. 
might suggest that physicians participating as COSEHC's members have higher skills and motivational capabilities to address the greater risk for cardiovascular events than their patient population is exposed to. Whether these lower rates are related to a more aggressive treatment approach in terms of use of specific medications, greater physician time spent with the subjects, or more frequent follow-up were not assessed in this study. Nevertheless, the programmatic structured educational system that COSEHC ${ }^{\mathrm{TM}}$ members are exposed to might be a factor. ${ }^{7,10,31}$

The changes in goal attainment in terms of SBP and lipids were the primary outcome metric used in our study. Sex differences in at-goal rates were also included in the analysis since accumulating data suggest important differences in response rates, medication use, and clinical outcomes between men and women. ${ }^{32-34}$ Several studies showed that the reduction of arterial pressure, particularly SBP, to the goals recommended by current and past guidelines leads to robust reductions in the occurrence of cardiovascular events. ${ }^{35-37}$ The focus of our study on SBP was determined by the nature of the factors that enter in the calculation of the COSEHCTM risk score, ${ }^{6,13}$ which derives further strength from studies showing a higher association of cardiovascular risk with systolic rather than diastolic blood pressure in subjects older than 50 years. ${ }^{31,38-41}$ A detailed analysis by Basile $^{42}$ documented the limitations of using response rates to a particular treatment rather than attainment of a predefined or recommended at-goal value. Our 33-month observational follow-up study in this large population demonstrates important sex differences in at-goal rates that become relatively less marked in diabetic subjects. Gueyffier et $\mathrm{al}^{32}$ reported that while antihypertensive treatment in men prevented as many coronary events as stroke events, a similar benefit of therapy in women was primarily reflected in a reduction in stroke rates. In our study, we showed that nondiabetic men evidenced higher at-goal rates for total and LDL-chol while lower at-goal rates occurred for SBP, HDL-chol, and plasma triglycerides. Furthermore, the logistic regression results demonstrated that goal rates for BMI had a much larger effect than sex. These findings do not negate the role of sex in the progression of CVD since a recent report showed a higher risk for large myocardial infarction and heart failure in females with the cardiometabolic syndrome. ${ }^{43}$

There were a number of limitations present in this study design. Since this was an observational study based on EMR data, patients have variable intervals between their baseline and follow-up visits. In some cases, data elements from multiple visits had to be combined to create a complete record.
The pharmaceutical records did not include stop dates, making it difficult to discern between patients adding additional therapies and patients switching therapies. Limitations of the COSEHCTM risk score are detailed elsewhere. ${ }^{6,13}$ The COSEHC $^{\mathrm{TM}}$ risk score provides a more accurate estimate of the absolute risk of cardiovascular events compared to the Framingham risk score, since the latter is based on a homogeneously white healthy individual population. Known limitations of the Framingham risk score include under (over) estimation of risk for non-Caucasian individuals (AfricanAmericans, Hispanic-Americans, and Native Americans). ${ }^{44,45}$ The wider generalizability and discriminatory sensitivity of the COSEHC ${ }^{\mathrm{TM}}$ risk score is based on the model published by the INdividual Data ANalysis of Antihypertensive intervention trials (INDANA) project, which is based on a much larger patient population of multiple ethnic origin. ${ }^{13,32,46-51}$

The interim analysis, 33 months after completion of baseline measures, provides new evidence about the difficulties that physicians face in improving treatment outcomes, particularly in terms of lipid management in women. There is clear evidence that diabetes worsens the ability of physicians to treat to goal, particularly in those subjects whose SBP and lipids were not at-goal rates at the completion of the baseline observational period. On the other hand, the data show improved control rates for SBP and even lipids in diabetic subjects who were or were not at-goal levels at the time of enrollment in the program. Our data further confirm previous studies showing that higher use of medications is required to control arterial pressure and that higher use of medications is associated with worse COSEHC ${ }^{\text {TM}}$-risk scores.

In summary, the GVRM Study documents the characteristics of risk factors' prevalence and response to treatment in a population at higher risk of cardiovascular events. At the time when the Centers for Medicare and Medicaid Services (CMS), as well as both small and large health care plans, strive to develop approaches to improve the quality and patient-centeredness of care for its enrollees, the studies and methods embedded in the GVRM Study allow for a quantitative assessment of absolute risk, based on a longitudinal monitoring of cardiovascular variables. While the practices participating in this project exercised their own independent best-practice approaches to the care of their patients, the regular reporting of cardiometabolic data captured from their electronic medical record systems with regular feedback to the providers make it plausible that these tools can contribute significantly to improved patient care and outcomes. 


\section{Acknowledgments}

Partial support for the conduction of this study was provided by grant 2PO1 HL-051952 from the National Heart, Lung and Blood Institute of the National Institutes of Health (CM Ferrario) and Daiichi Sankyo, Inc. We thank Alex Sheek of COSEHC ${ }^{\text {TM }}$ for technical assistance and the following COSEHC Centers of Excellence ${ }^{\mathrm{TM}}$ investigators for participating in the GVRM Study: Bryan N Batson of the Hattiesburg Clinic, Hattiesburg, MS, USA; Michael Canfield, formerly of the Palmetto Primary Care Physicians, North Charleston, SC, USA; Alexander Cohen, of Pee Dee Healthcare, Darlington, SC, USA; Gary Goforth, formerly of Self Regional Healthcare, Greenwood, SC, USA; Mark Houston, of the Hypertension Institute at St Thomas Hospital, Nashville, TN, USA; and Steve Ross, of Internal Medicine Specialists, Florence, SC, USA.

\section{Disclosure}

The authors report no conflicts of interest in this work.

\section{References}

1. Athyros VG, Ganotakis ES, Elisaf MS, Liberopoulos EN, Goudevenos IA, Karagiannis A; GREECE-METS Collaborative Group. Prevalence of vascular disease in metabolic syndrome using three proposed definitions. Int J Cardiol. 2007;117:204-210.

2. Daskalopoulou SS, Athyros VG, Kolovou GD, Anagnostopoulou KK, Mikhailidis DP. Definitions of metabolic syndrome: Where are we now? Curr Vasc Pharmacol. 2006;4(3):185-197.

3. de Simone G, Devereux RB, Chinali M, et al; Strong Heart Study Investigators. Prognostic impact of metabolic syndrome by different definitions in a population with high prevalence of obesity and diabetes: the Strong Heart Study. Diabetes Care. 2007;30(7):1851-1856.

4. Dekker JM, Girman C, Rhodes T, et al. Metabolic syndrome and 10-year cardiovascular disease risk in the Hoorn Study. Circulation. 2005;112(5):666-673.

5. Gami AS, Witt BJ, Howard DE, et al. Metabolic syndrome and risk of incident cardiovascular events and death: a systematic review and meta-analysis of longitudinal studies. J Am Coll Cardiol. 2007;49(4):403-414.

6. Ferrario CM, Moore MA, Bestermann W Jr, et al. COSEHC global vascular risk management quality improvement program: rationale and design. Vasc Health Risk Manag. 2010;6:1135-1145.

7. Bestermann W, Houston MC, Basile J, et al. Addressing the global cardiovascular risk of hypertension, dyslipidemia, diabetes mellitus, and the metabolic syndrome in the southeastern United States, part II: treatment recommendations for management of the global cardiovascular risk of hypertension, dyslipidemia, diabetes mellitus, and the metabolic syndrome. Am J Med Sci. 2005;329(6): 292-305.

8. Egan BM, Lackland DT, Igho-Pemu P, et al. Cardiovascular risk factor control in communities - update from the ASH CarolinasGeorgia Chapter, the Hypertension Initiative, and the Community Physicians' Network. J Clin Hypertens (Greenwich). 2006;8(12): 879-886.

9. Ferrario CM. Highlights of the Tenth Annual COSEHC National Scientific Session. Am J Med Sci. 2004;327(5):234-235.

10. Ferrario CM. COSEHC overview. Am J Med Sci. 2004;327(5):233.

11. Ferrario CM. Highlights of the Twelfth Annual COSEHC National Scientific Session. Am J Med Sci. 2006;331(2):59.
12. Hall WD, Ferrario CM, Moore MA, et al. Hypertension-related morbidity and mortality in the southeastern United States. Am J Med Sci. 1997;313(4):195-209.

13. Houston MC, Basile J, Bestermann WH, et al. Addressing the global cardiovascular risk of hypertension, dyslipidemia, and insulin resistance in the southeastern United States. Am J Med Sci. 2005;329(6):276-291.

14. Jones D, Basile J, Cushman W, et al. Managing hypertension in the southeastern United States: applying the guidelines from the Sixth Report of the Joint National Committee on Prevention, Detection, Evaluation, and Treatment of High Blood Pressure (JNC VI). Am J Med Sci. 1999;318(6):357-364.

15. Lackland DT, Bendall HE, Osmond C, Egan BM, Barker DJ. Low birth weights contribute to high rates of early-onset chronic renal failure in the Southeastern United States. Arch Intern Med. 2000;160(10):1472-1476.

16. National Cholesterol Education Program (NCEP) Expert Panel on Detection, Evaluation, and Treatment of High Blood Cholesterol in Adults (Adult Treatment Panel III). Third Report of the National Cholesterol Education Program (NCEP) Expert Panel on Detection, Evaluation, and Treatment of High Blood Cholesterol in Adults (Adult Treatment Panel III) final report. Circulation. 2002;106(25):3143-3421.

17. Chobanian AV, Bakris GL, Black HR, et al; National Heart, Lung, and Blood Institute Joint National Committee on Prevention, Detection, Evaluation, and Treatment of High Blood Pressure; National High Blood Pressure Education Program Coordinating Committee. The Seventh Report of the Joint National Committee on Prevention, Detection, Evaluation, and Treatment of High Blood Pressure: the JNC 7 report. JAMA. 2003;289(19):2560-2572.

18. Go AS, Mozaffarian D, Roger VL, et al; American Heart Association Statistics Committee and Stroke Statistics Subcommittee. Executive summary: heart disease and stroke statistics - 2013 update: a report from the American Heart Association. Circulation. 2013;127(1):143-152.

19. Howard VJ, Woolson RF, Egan BM, et al. Prevalence of hypertension by duration and age at exposure to the stroke belt. J Am Soc Hypertens. 2010;4(1):32-41.

20. Lackland DT, Egan BM. The dominant role of systolic hypertension as a vascular risk factor: evidence from the southeastern United States. Am J Med Sci. 1999;318(6):365-368.

21. AIM-HIGH Investigators; Boden WE, Probstfield JL, Anderson T, et al. The role of niacin in raising high-density lipoprotein cholesterol to reduce cardiovascular events in patients with atherosclerotic cardiovascular disease and optimally treated low-density lipoprotein cholesterol Rationale and study design. The Atherothrombosis Intervention in Metabolic syndrome with low HDL/high triglycerides: Impact on Global Health outcomes (AIM-HIGH). Am Heart J. 2011;161(3):471-477.

22. AIM-HIGH Investigators; Boden WE, Probstfield JL, Anderson T, et al. Niacin in patients with low HDL cholesterol levels receiving intensive statin therapy. $N$ Engl J Med. 2011;365(24):2255-2267.

23. Dehghan M, Mente A, Teo KK, et al. Relationship between healthy diet and risk of cardiovascular disease among patients on drug therapies for secondary prevention: a prospective cohort study of 31546 high-risk individuals from 40 countries. Circulation. 2012;126(23): 2705-2712.

24. Michos ED, Sibley CT, Baer JT, Blaha MJ, Blumenthal RS. Niacin and statin combination therapy for atherosclerosis regression and prevention of cardiovascular disease events: reconciling the AIMHIGH (Atherothrombosis Intervention in Metabolic Syndrome With Low HDL/High Triglycerides: Impact on Global Health Outcomes) trial with previous surrogate endpoint trials. J Am Coll Cardiol. 2012;59(23):2058-2064.

25. Teo K, Yusuf S, Sleight P, et al; ONTARGET/TRANSCEND Investigators. Rationale, design, and baseline characteristics of 2 large, simple, randomized trials evaluating telmisartan, ramipril, and their combination in high-risk patients: the Ongoing Telmisartan Alone and in Combination with Ramipril Global Endpoint Trial/Telmisartan Randomized Assessment Study in ACE Intolerant Subjects with Cardiovascular Disease (ONTARGET/TRANSCEND) trials. Am Heart J. 2004;148(1):52-61. 
26. Teo KK, Mitchell LB, Pogue J, Bosch J, Dagenais G, Yusuf S; HOPE Investigators. Effect of ramipril in reducing sudden deaths and nonfatal cardiac arrests in high-risk individuals without heart failure or left ventricular dysfunction. Circulation. 2004;110:1413-1417.

27. McQueen MJ, Kavsak PA, Xu L, Shestakovska O, Yusuf S. Predicting myocardial infarction and other serious cardiac outcomes using highsensitivity cardiac troponin $\mathrm{T}$ in a high-risk stable population. Clin Biochem. 2013;46(1-2):5-9.

28. ONTARGET Investigators; Yusuf S, Teo KK, Pogue J, et al. Telmisartan, ramipril, or both in patients at high risk for vascular events. N Engl J Med. 2008;358(15):1547-1559.

29. Telmisartan Randomised AssessmeNt Study in ACE iNtolerant subjects with cardiovascular Disease (TRANSCEND) Investigators; Yusuf S, Teo K, Anderson C, et al. Effects of the angiotensin-receptor blocker telmisartan on cardiovascular events in high-risk patients intolerant to angiotensin-converting enzyme inhibitors: a randomised controlled trial. Lancet. 2008;372(9644):1174-1183.

30. Yusuf S, Diener HC, Sacco RL, et al; PRoFESS Study Group. Telmisartan to prevent recurrent stroke and cardiovascular events. N Engl J Med. 2008;359(12):1225-1237.

31. Basile J, Houston M, Ferrario C. Incremental risk-factor reduction improves overall cardiovascular benefit: is it time to abandon the silos? J Clin Hypertens (Greenwich). 2006;8(10):686-688.

32. Gueyffier F, Boutitie F, Boissel JP, et al. Effect of antihypertensive drug treatment on cardiovascular outcomes in women and men. A meta-analysis of individual patient data from randomized, controlled trials. The INDANA Investigators. Ann Intern Med. 1997;126(10): 761-767.

33. Jones CA, Nagpal S. An update: women, hypertension and therapeutic efficacy. Can J Cardiol. 2001;17(12):1283-1289.

34. Quan A, Kerlikowske K, Gueyffier F, Boissel JP; INDANA investigators. Pharmacotherapy for hypertension in women of different races. Cochrane Database Syst Rev. 2000;(3):CD002146.

35. Mancia G, Grassi G. Antihypertensive treatment: past, present and future. J Hypertens Suppl. 1998;16(1):S1-S7.

36. Zanchetti A, Mancia G, Black HR, et al. Facts and fallacies of blood pressure control in recent trials: implications in the management of patients with hypertension. $J$ Hypertens. 2009;27(4):673-679.

37. Zanchetti A, Grassi G, Mancia G. When should antihypertensive drug treatment be initiated and to what levels should systolic blood pressure be lowered? A critical reappraisal. J Hypertens. 2009;27(5):923-934.

38. Bestermann WH, Lackland DT, Riehle JE, Egan BM. A systematic approach to managing hypertension and the metabolic syndrome in primary care. South Med J. 2004;97(10):932-938.
39. Mancia G, Omboni S, Parati G. The importance of blood pressure variability in hypertension. Blood Press Monit. 2000;5 Suppl 1:S9-S15.

40. Mancia G, Grassi G. Systolic and diastolic blood pressure control in antihypertensive drug trials. J Hypertens. 2002;20(8):1461-1464.

41. Mancia G. Defining blood pressure goals: is it enough to manage total cardiovascular risk? J Hypertens Suppl. 2009;27(5):S3-S8.

42. Basile J. Blood pressure responder rates versus goal rates: which metric matters? Ther Adv Cardiovasc Dis. 2009;3(2):157-174.

43. Kranjeec D, Altabas V. Metabolic syndrome influencing infarct size and heart failure in patients with acute coronary syndrome: does gender matter? Endocr J. 2012;59(12):1065-1076.

44. Pocock SJ, McCormack V, Gueyffier F, Boutitie F, Fagard RH, Boissel JP. A score for predicting risk of death from cardiovascular disease in adults with raised blood pressure, based on individual patient data from randomised controlled trials. BMJ. 2001;323(7304):75-81.

45. Schlendorf KH, Nasir K, Blumenthal RS. Limitations of the Framingham risk score are now much clearer. Prev Med. 2009;48(2):115-116.

46. Gueyffier F, Boutitie F, Boissel JP, et al. INDANA: a meta-analysis on individual patient data in hypertension. Protocol and preliminary results. Therapie. 1995;50(4):353-362.

47. Gueyffier F, Boissel JP, Boutitie F, et al. Effect of antihypertensive treatment in patients having already suffered from stroke. Gathering the evidence. The INDANA (INdividual Data ANalysis of Antihypertensive intervention trials) Project Collaborators. Stroke. 1997;28(12):2557-2562.

48. Gueyffier F, Bulpitt C, Boissel JP, et al. Antihypertensive drugs in very old people: a subgroup meta-analysis of randomised controlled trials. INDANA Group. Lancet. 1999;353(9155):793-796.

49. Li W, Gueyffier F, Boissel JP, Girard P, Boutitie F, Cucherat M. [Identification and prediction of responders to a therapy. A model and its preliminary application to hypertension.] Arch Mal Coeur Vaiss. 1998;91(8):1059-1063. French [with English abstract].

50. Lièvre M, Gueyffier F, Ekbom T, et al. Efficacy of diuretics and betablockers in diabetic hypertensive patients. Results from a meta-analysis. The INDANA Steering Committee. Diabetes Care. 2000;23 Suppl 2: B65-B71.

51. Matsumoto M. Cerebrovascular disease: [Impact of INDANA meta-analysis and PROGRESS trial.] Nippon Rinsho. 2004;62 Suppl 3: 605-611. Japanese.
Vascular Health and Risk Management

\section{Publish your work in this journal}

Vascular Health and Risk Management is an international, peerreviewed journal of therapeutics and risk management, focusing on concise rapid reporting of clinical studies on the processes involved in the maintenance of vascular health; the monitoring, prevention and treatment of vascular disease and its sequelae; and the involvement of

\section{Dovepress}

metabolic disorders, particularly diabetes. This journal is indexed on PubMed Central and MedLine. The manuscript management system is completely online and includes a very quick and fair peer-review system, which is all easy to use. Visit http://www.dovepress.com/ testimonials.php to read real quotes from published authors. 